\title{
Correcting direction-dependent gains in the deconvolution of radio interferometric images
}

\author{
S. Bhatnagar ${ }^{1}$, T. J. Cornwell ${ }^{2}$, K. Golap ${ }^{1}$, and J. M. Uson ${ }^{3}$ \\ ${ }^{1}$ National Radio Astronomy Observatory ${ }^{\star}, 1003$ Lopezville Road, Socorro, NM, 87801, USA \\ e-mail: [sbhatnag;kgolap]@nrao.edu \\ 2 Australia Telescope National Facility, Epping, New South Wales, Australia, 2120, Australia \\ e-mail: Tim.Cornwell@csiro.au \\ 3 National Radio Astronomy Observatory*, 520 Edgemont Road, Charlottesville, VA, 22903, USA \\ e-mail: juson@nrao.edu \\ Received 19 December 2007 / Accepted 3 June 2008
}

ABSTRACT

\begin{abstract}
Astronomical imaging using aperture synthesis telescopes requires deconvolution of the point spread function as well as calibration of instrumental and atmospheric effects. In general, such effects are time-variable and vary across the field of view as well, resulting in direction-dependent (DD), time-varying gains. Most existing imaging and calibration algorithms assume that the corruptions are direction independent, preventing even moderate dynamic range full-beam, full-Stokes imaging. We present a general framework for imaging algorithms which incorporate DD errors. We describe as well an iterative deconvolution algorithm that corrects known DD errors due to the antenna power patterns (including errors due to the antenna polarization response) as well as pointing errors for high dynamic range full-beam polarimetric imaging. Using simulations we demonstrate that errors due to realistic primary beams as well as antenna pointing errors will limit the dynamic range of upcoming higher sensitivity instruments like the EVLA and ALMA and that our new algorithm can be used to correct for such errors. We show that the technique described here corrects for effects that can be described as approximate unitary operators in the interferometric measurement equation, such as those due to antenna pointing errors and non-azimuthally symmetric antenna power patterns. We have applied this algorithm to VLA $1.4 \mathrm{GHz}$ observations of a field that contains two "4C" sources and have obtained Stokes $I$ and $V$ images with systematic errors that are one order of magnitude lower than those obtained with conventional imaging tools. Residual systematic errors that are seen at a level slightly above that of the thermal noise are likely due to selfcalibration instabilities that are triggered by a combination of unknown pointing errors and errors in our assumption of the shape of the primary beam of each antenna. We hope to present a more refined algorithm to deal with the fully general case in due course. Our simulations show that on data with no other calibration errors, the algorithm corrects pointing errors as well as errors due to known asymmetries in the antenna pattern.
\end{abstract}

Key words. methods: data analysis - techniques: interferometic - techniques: image processing - techniques: polarimetric

\section{Introduction}

Astronomical observations made with interferometric radio telescopes suffer from variable gain effects which can be broadly classified as direction-independent (DI) and directiondependent (DD) errors. The complex instrumental gains due to the electronic devices that follow the feed elements are directionally independent, whereas the time-varying gains due to the antenna primary beams provide an example of direction-dependent gains. Direction-independent effects may be corrected separately from imaging, so most processing algorithms have been limited to treating such effects. Direction-dependent gains are more difficult to incorporate since they must be applied during imaging which has slowed progress thus far. This must now change, however, because direction dependent gains are expected to limit observations with existing as well as next generation telescopes presently under construction. Indeed, a number of deep observations made with present telescopes have been limited already by direction-dependent, time-variable errors. In this, the first of two papers, we present an algorithmic framework that allows incorporation of a class of directionally dependent gain effects during

* Associated Universities Inc. operates the National Radio Astronomy Observatory under cooperative agreement with the National Science Foundation. deconvolution. A key part of this framework is the provision of an efficient transform between the data and image domains. As an example of the application of this framework, we demonstrate that the effects of known antenna pointing errors and beam polarization can be corrected during imaging. We also discuss error propagation and the required computing resources. A second paper will describe an algorithm constructed within this framework that allows solving for parameters that describe such gain changes.

\section{The measurement equation}

The measurement equation that describes astronomical imaging using aperture synthesis telescopes can be compactly written using the Hamaker-Bregman-Sault notation (Hamaker et al. 1996) as ${ }^{1}$ :

$V_{i j}^{\mathrm{Obs}}=\mathrm{M}_{i j} \int \mathrm{M}_{i j}^{\mathrm{Sky}}(\boldsymbol{s}) I(\boldsymbol{s}) \mathrm{e}^{2 \pi \imath s \cdot b_{i j}} \mathrm{~d} \boldsymbol{s}$

\footnotetext{
${ }^{1}$ All expressions in this paper are in the signal-domain polarization frame (the linear or circular polarization bases). Conversion to and from the Stokes frame can be done by the application of an appropriate coordinate transform operator (Hamaker et al. 1996). Note that only the minor cycle of the deconvolution iterations operate in the Stokes frame (see Sect. 4).
} 
where $V_{i j}^{\text {Obs }}$ is the observed full polarization visibility vector, $\mathrm{M}_{i j}$ and $\mathrm{M}_{i j}^{\text {Sky }}(s)$ are the Mueller Matrices (Mueller 1948) for DI and DD gains respectively, $s$ is a direction in the sky, $I$ is the image and $\boldsymbol{b}_{i j}$ is the vector that describes the projected separation between the antennas $i$ and $j$ in units of the wavelength of the observation.

Measurements sample this equation, providing constraints on the unknowns on the right hand side. The sky brightness distribution $I(s)$ has to be estimated in the presence of known or unknown gain terms $\mathrm{M}_{i j}$ and $\mathrm{M}_{i j}^{\mathrm{Sky}}$. However, the measurement equation cannot simply be inverted as it is not a Fourier transform. Furthermore, as is the case for simpler forms of the measurement equation, the visibility is sampled only at a limited set of points so there is insufficient information to determine the solution exactly. We follow the normal terminology and call the estimation of the sky brightness "deconvolution" though it is only vaguely related to the typical deconvolution of a shift-invariant point-spread function (Andrews \& Hunt 1977). The other part of imaging is the (necessary) estimation and correction of the gains either assuming that the sky brightness distribution is known ("calibration") or determined simultaneously ("self-calibration").

Most often, deconvolution and self-calibration are performed using simple steepest-descent algorithms. The residuals are minimized in the least square sense by minimizing $\chi^{2}$ which can be expressed in terms of the data $(\boldsymbol{V})$ and the model data $\left(\boldsymbol{V}^{M}\right)$ as

$\chi^{2}=\left[\boldsymbol{V}-\boldsymbol{V}^{M}\right]^{\dagger} \Lambda\left[\boldsymbol{V}-\boldsymbol{V}^{M}\right]$

where $\Lambda$ is the inverse of the measurement noise covariance matrix. The sky-brightness model, the unknown gain terms as well as suitable imaging weights are included in $\boldsymbol{V}^{M}$. Gradients of $\chi^{2}$ with respect to the sky brightness model and the gain terms may be calculated straightforwardly and used in an iterative minimization algorithm to solve for the sky brightness model and unknown gains (Schwarz 1978; Bhatnagar \& Cornwell 2004). Often, this results in complex and non-linear algorithms but these seem to work well most of the time. To make a practical algorithm, it is necessary to find efficient ways of calculating the gradients of $\chi^{2}$ with respect to the unknowns. In this paper, we consider the case of estimating the sky brightness in the presence of known directionally dependent gain terms. In a subsequent paper, we will consider the estimation of parameters describing unknown gain terms.

There are cases in which the deconvolution and correction for the Mueller matrix can be decoupled. For example, direction dependent effects which are identical for all the measurements can be removed by dividing the deconvolved image by $M^{\text {Sky }}$. The correction for an azimuthally symmetric and time-constant antenna power pattern provides one such example. The deconvolution is then performed on the entire data set, ignoring the antenna power pattern, whose inverse function is applied to the image only after the deconvolution and self-calibration have been completed. However, this assumption often breaks down. For example, the antenna power pattern for most practical antenna geometries is azimuthally asymmetric (because of off-axis feed position, asymmetric subreflector, feed-legs, ...) and rotates on the sky for azimuth-elevation mount telescopes resulting in offaxis gains which vary with parallactic angle (PA). In such case, processing time slices of the data independently might lower the range of the gain variations in each subset. The final deconvolved images for each subset are averaged post-deconvolution. This is straightforward and often used, but can be expected to be sub-optimal because the deconvolution step is inherently nonlinear and higher PSF sidelobes for individual subsets increase the level of (non-symmetric) deconvolution errors in each subimage. Hence it would seem preferable to follow a procedure that applied the corrections while imaging the full data set.

\section{Example of directionally dependent gains}

In this section, we consider in detail an example of directionally dependent gains - the antenna far-field voltage pattern.

The far-field voltage pattern is the Fourier transform of the antenna illumination function (Kraus 1986). Thus it is typically the case that because of the details of the antenna geometry (such as quadrupod legs) and feed design, the antenna voltage patterns are azimuthally asymmetric. Furthermore, the polarization response of the antenna will vary away from the antenna optical axis due to antenna geometry and the physics of the reflection of electromagnetic waves from curved surfaces. In addition, as an interferometric array composed of altitude-elevation mounted antennas tracks a region of the sky, these asymmetrical antenna voltage patterns rotate on the sky. This, along with significant time varying antenna pointing errors, makes $\mathrm{M}^{\text {Sky }}$ time varying and different for each antenna pair (interferometric baseline). Even equatorially mounted antennas share in this problem to the extent that changes in elevation (temperature) might deform the antennas due to gravity (dilation).

\subsection{The Jones and Mueller matrices}

The Mueller matrix is an outer product of the two antenna based Jones matrices (Jones 1941; Hamaker et al. 1996). A full direction-dependent polarimetric description requires a Jones matrix per pixel in the image. For the two orthogonal polarizations, labeled $p$ and $q$, the Sky Jones matrix as a function of direction is given by:

$$
\mathrm{J}_{i}^{\mathrm{Sky}}(\boldsymbol{s})=\left[\begin{array}{cc}
\mathrm{J}_{i}^{p} & -\mathrm{J}_{i}^{p q} \\
\mathrm{~J}_{i}^{q p} & \mathrm{~J}_{i}^{q}
\end{array}\right] .
$$

The super-scripts $p q$ and $q p$ represent leakage of the $q$-polarization signal to $p$-polarization signal and vice-versa. The diagonal elements correspond to the antenna voltage patterns on the sky while the off-diagonal elements correspond to the polarization leakage terms ( $p \rightarrow q$ and $q \rightarrow p$ ) due to instrumental leakage (antenna geometry, electronics) and/or atmospheric, ionospheric or other transmission effects such as Faraday rotation.

The full direction-dependent Sky Mueller matrix $M_{i j}^{\text {Sky }}$ for baseline $i-j$ is a $4 \times 4$ matrix:

$$
\begin{aligned}
\mathrm{M}_{i j}^{\mathrm{Sky}}(s) & =\mathrm{J}_{i}^{\mathrm{Sky}}(\boldsymbol{s}) \otimes \mathrm{J}_{j}^{\mathrm{Sky}}(s) \\
& =\left[\begin{array}{cccc}
\mathrm{J}_{i}^{p} \mathrm{~J}_{j}^{p^{*}} & -\mathrm{J}_{i}^{p} \mathrm{~J}_{j}^{p q^{*}} & -\mathrm{J}_{i}^{p q} \mathrm{~J}_{j}^{p^{*}} & \mathrm{~J}_{i}^{p q} \mathrm{~J}_{j}^{p q^{*}} \\
\mathrm{~J}_{i}^{p} \mathrm{~J}_{j}^{q p^{*}} & \mathrm{~J}_{i}^{p} \mathrm{~J}_{j}^{q^{*}} & -\mathrm{J}_{i}^{p q} \mathrm{~J}_{j}^{q p^{*}} & -\mathrm{J}_{i}^{p q} \mathrm{~J}_{j}^{q^{*}} \\
\mathrm{~J}_{i}^{q p} \mathrm{~J}_{j}^{p^{*}} & -\mathrm{J}_{i}^{q p} \mathrm{~J}_{j}^{p q^{*}} & \mathrm{~J}_{i}^{q} \mathrm{~J}_{j}^{p^{*}} & -\mathrm{J}_{i}^{q} \mathrm{~J}_{j}^{p q^{*}} \\
\mathrm{~J}_{i}^{q p} \mathrm{~J}_{j}^{q p^{*}} & \mathrm{~J}_{i}^{q p} \mathrm{~J}_{j}^{q^{*}} & \mathrm{~J}_{i}^{q} \mathrm{~J}_{j}^{q p^{*}} & \mathrm{~J}_{i}^{q} \mathrm{~J}_{j}^{q^{*}}
\end{array}\right] .
\end{aligned}
$$

The diagonal elements of this matrix are the antenna power patterns for the four polarization products, whereas the off-diagonal products incorporate the cross-polarization leakage terms. For the VLA antennas, where the two circular polarization power 

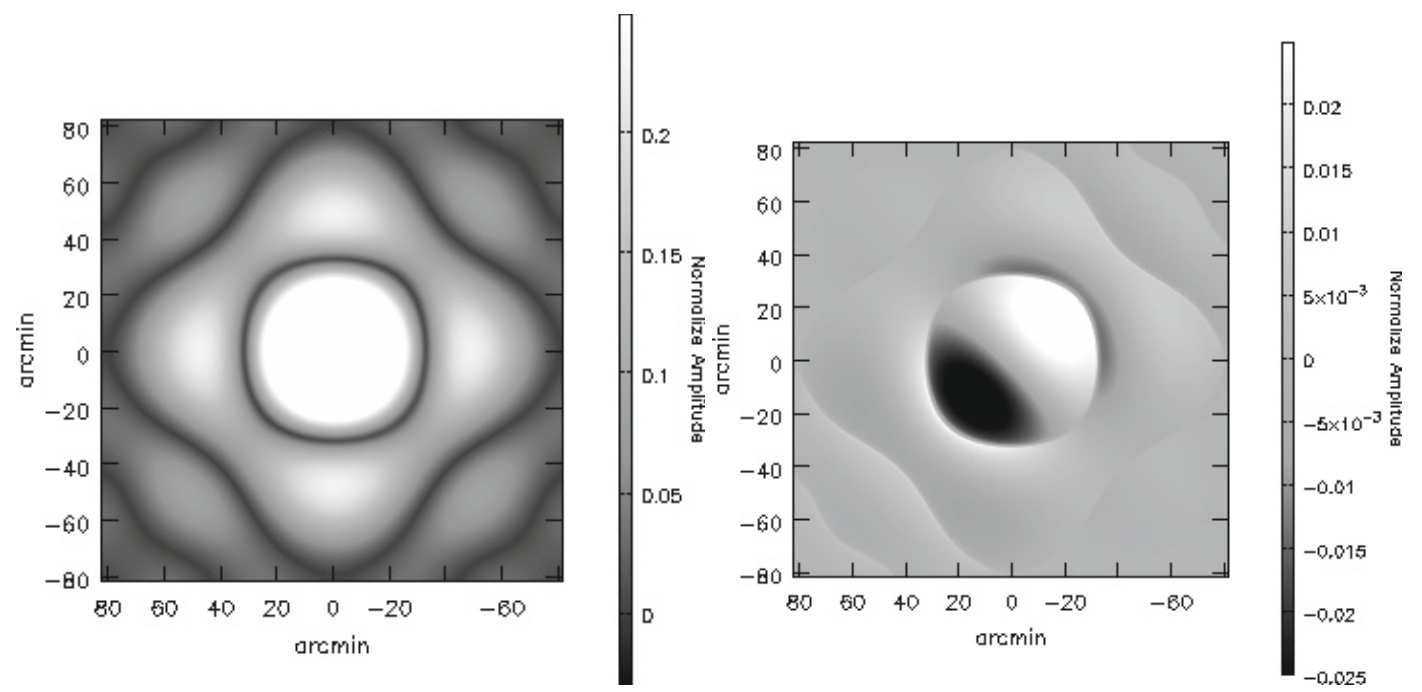

Fig. 1. The image in the left panel shows a typical (first) diagonal term for the VLA at $1.4 \mathrm{GHz}\left(\mathrm{J}_{i}^{\mathrm{R}} \mathrm{J}_{j}^{\mathrm{R}^{*}}\right)$ of the Sky Mueller matrix. The pattern is off-center due to the off-axis location of the feeds. The image in the right panel shows the difference between the first and second diagonal term $\left(\mathrm{J}_{i}^{\mathrm{R}} \mathrm{J}_{j}^{\mathrm{R}^{*}}-\mathrm{J}_{i}^{\mathrm{R}} \mathrm{J}_{j}^{\mathrm{L}^{*}}\right)$. $\mathrm{J}^{\mathrm{Sky}}$ was evaluated at PA $=0$ and normalized by the peak of one of the diagonal terms (the peak values of both diagonal terms are the same).
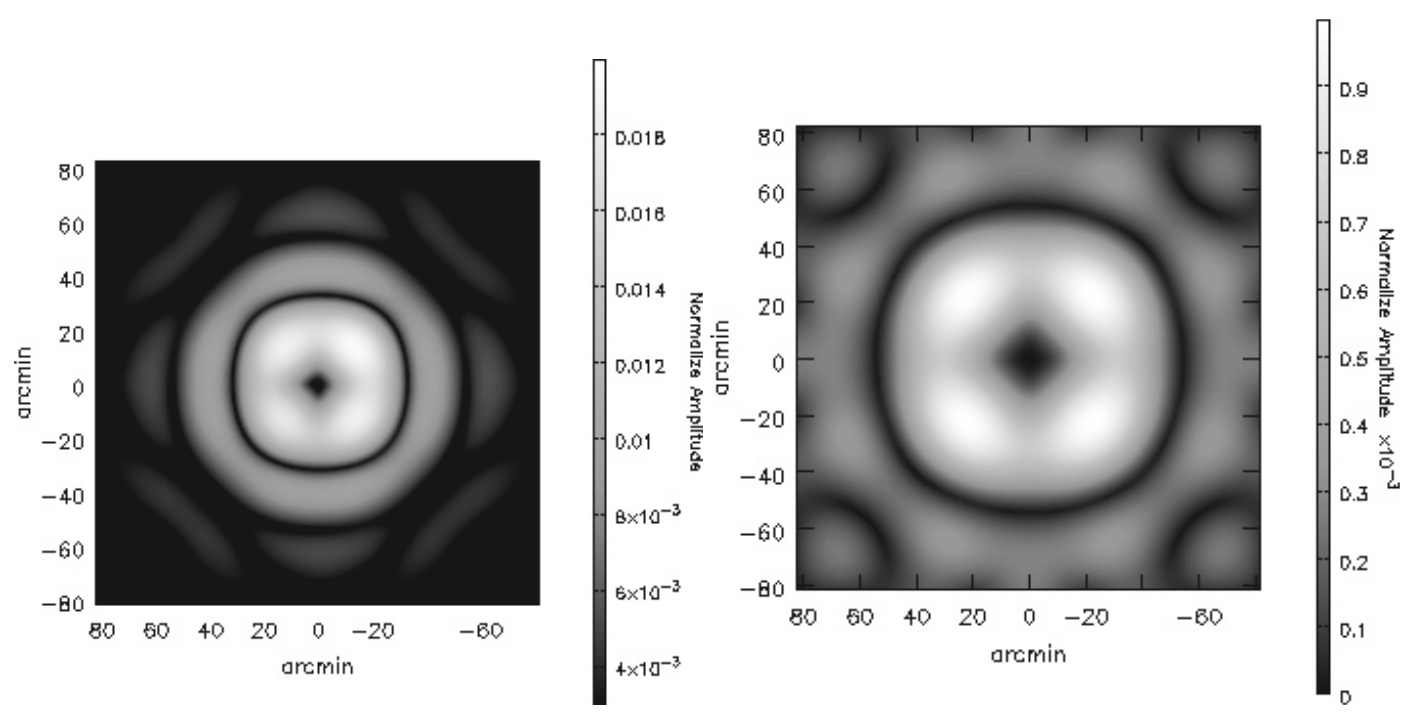

Fig. 2. The off-diagonal terms of the Sky Mueller matrix for VLA antennas at $1.4 \mathrm{GHz}$. The image in the left panel is of first order in the antenna leakage $\left(\mathrm{J}_{i}^{\mathrm{R}} \mathrm{J}_{j}^{\mathrm{RL}}\right)$. The image in the right panel is the second order term in antenna leakage $\left(\mathrm{J}_{i}^{\mathrm{RL}} \mathrm{JL}_{j}^{\mathrm{RL}}\right)$. $\mathrm{J}^{\mathrm{Rky}}$ was evaluated at PA $=0$ and normalized by the peak of one of the diagonal terms (the peak values of both diagonal terms are the same).

patterns are squinted with respect to each other, the power pattern for the parallel hand $\left(J_{i}^{\mathrm{R}} J_{j}^{\mathrm{R}^{*}}\right)$ and the difference between a parallel hand and a cross hand product $\left(\mathrm{J}_{i}^{\mathrm{R}} \mathrm{J}_{j}^{\mathrm{R}^{*}}-\mathrm{J}_{i}^{\mathrm{R}} \mathrm{J}_{j}^{\mathrm{L}^{*}}\right)$ are shown in Fig. 1 (super-script $R$ and $L$ denotes the right- and leftcircular polarizations respectively). The main lobe of the power pattern is azimuthally asymmetric and, clearly, highly asymmetric in the first sidelobe. This asymmetry is due to aperture blockage by the feed and the feed-legs. The two parallel-hand power patterns $\left(\mathrm{J}_{i}^{\mathrm{R}} \mathrm{J}_{j}^{\mathrm{R}^{*}}\right.$ and $\mathrm{J}_{i}^{\mathrm{L}} \mathrm{J}_{j}^{L^{*}}$ diagonal terms) are also not identical because of differences between the power patterns for the two orthogonal polarizations. Rotation of these patterns on the sky as a function of PA leads to time varying, direction-dependent gains. Even differences between two diagonal terms, for example $\mathrm{J}_{i}^{\mathrm{R}} \mathrm{J}_{j}^{\mathrm{R}^{*}}-\mathrm{J}_{i}^{\mathrm{R}} \mathrm{J}_{j}^{\mathrm{L}^{*}}$, are on the order of a few percent and vary with position within the beam. In addition, differences between antennas (focus, surface accuracy, pointing) will lead to secondorder differences in the values of any given term. Therefore, an assumption of diagonal form for $\mathrm{M}_{i j}^{\mathrm{sky}}$ will lead to errors in the image domain - particularly in the presence of strong sources located in the outer parts of the main lobe as well as in the first few sidelobes.

For high dynamic range imaging $(\geq \mathrm{few} \times 1000)$, the offdiagonal terms of the Mueller matrix are non-negligible, vary across the entire beam and, typically, increase substantially with distance from the center. Figure 2 shows typical off-diagonal terms for the VLA (the cross polar power patterns $\mathrm{J}_{i}^{\mathrm{R}} \mathrm{J}_{j}^{\mathrm{RL} *}$ and $\left.J_{i}^{\mathrm{RL}} \mathrm{J}_{j}^{\mathrm{R} L^{*}}\right)$. These are the higher order leakage terms and are purely due to the leakage of the orthogonal polarization signals into the complementary polarization in the signal path from various antennas The term shown in the left-hand-side panel is the 
first order leakage term and has peak amplitude relative to the diagonal terms of $\sim 10^{-2}$. Since this is comparable to the difference between the diagonal terms, ignoring this term will result in imaging artifacts similar to those due to the assumption that parallel- and cross-hand power patterns are identical. The second order term $\mathrm{J}_{i}^{\mathrm{RL}} \mathrm{J}_{j}^{\mathrm{RL}}{ }^{*}$, shown in the right hand side panel, has an amplitude of $\sim 10^{-4}$. Consequently, for high dynamic range imaging $(\geq$ few $\times 1000)$, the $\mathrm{M}_{i j}^{\text {Sky }}$ cannot be approximated as even a diagonally dominant matrix. For even moderate dynamic range full-Stokes, full-beam imaging, this difference needs to be taken into account.

Existing calibration procedures split the errors into two antenna based Jones matrices - the $G$ and $D$ matrices for complex gain and polarization leakage, respectively. $G$ is assumed to be purely diagonal while $D$ is unity along the diagonal and the offdiagonal terms are the leakage gains (Hamaker et al. 1996). The directional dependence of these terms is ignored and the values of the complex gains and leakage gains at the center of the field are used throughout the beam. Typically, polarization leakage is small near the optical axis of the antenna. Hence, for imaging compact sources at the center of the beam, the Sky Mueller matrix is diagonally dominant and the above approximation is justified. When imaging fields with significant emission throughout the primary beam, this approximation will lead to artifacts and significantly lower image fidelity away from the image center. This is even more true for the case of mosaic imaging (Cornwell 1988) where there is significant flux density throughout the primary beam for most pointings.

Therefore for high dynamic range full beam imaging, full treatment of polarization has to be kept in the entire imaging and calibration process. We refer to this as J-Matrix based imaging and calibration. Stokes images have to be made from the linear addition of the visibilities from all polarization products, weighted by the appropriate terms of the Sky Mueller matrix. Strictly speaking, even conventional Stokes-I imaging using only the parallel hand visibilities is incorrect. For moderate dynamic range full beam, full Stokes imaging, it may be possible to use only the diagonal terms of the Sky Mueller matrix. Note that the computational load of J-Matrix based imaging is a factor of four higher than the corresponding load for conventional imaging (where the J-Matrix is assumed to be purely diagonal).

\section{A deconvolution algorithm incorporating direction-dependent gain correction}

As described above, most iterative deconvolution algorithms derive updates $\left(\Delta \boldsymbol{I}^{M}\right)$ to the existing model image $\left(\boldsymbol{I}^{M}\right)$ from the gradients of chi-square with respect to the unknown sky brightness:

$\Delta \boldsymbol{I}^{M}=-\mathrm{C} \partial \chi^{2} / \partial \boldsymbol{I}^{M}=\mathrm{C} \boldsymbol{I}^{R}$

where $\mathrm{C}$ is a scaling term, either a constant or the inverse Hessian (or an approximation thereof).

Typically, the model image is iteratively improved as:

$\boldsymbol{I}_{i}^{M}=\mathrm{T}\left(\boldsymbol{I}_{i-1}^{M},\left[\boldsymbol{I}_{i}^{R}\right]\right)$

where $\boldsymbol{I}_{i}^{R}$ is the Fourier transform of the residual visibilities $\left(\boldsymbol{V}^{R}\right)$ and $\boldsymbol{I}_{i}^{M}$ is the cumulative model at the $i$ th iteration. The operator $\mathrm{T}$ selects part of the gradient image. For the Högbom Clean algorithm (Högbom 1974), T simply updates the model by adding a scaled version of the peak in the image.
Following terminology established by Clark (1980), the calculation of the derivative for a given estimate is called the major cycle, and the application of the T operator is called the minor cycle. The minor cycle typically operates in the Stokes frame. The operator $T$ includes conversions between the signal-domain polarization frame and the Stokes frame using an appropriate coordinate transform operator (Hamaker et al. 1996).

The major cycle can be broken into two calculations:

forward: In the forward step, the model visibilities for baseline $i-j$ are calculated from the existing model image $\boldsymbol{I}^{M}$, using the equation:

$$
\boldsymbol{V}^{M}=\mathrm{A} \boldsymbol{I}^{M}
$$

where the operator $A$ is the measurement matrix.

backward: In the backward step, the residual visibilities $\left(\boldsymbol{V}^{R}\right)$ are propagated backwards to the image plane using the equation:

$$
\boldsymbol{I}^{R}=\left[\mathrm{A}^{\dagger} \mathrm{A}\right]^{-1} \mathrm{~A}^{\dagger} \boldsymbol{V}^{R} \text {. }
$$

The forward calculation must be done with high accuracy, but since the overall approach is iterative, the backward calculation may be performed with lower accuracy (Schwab 1983; Cotton 1999) when using the FFT algorithm for computing the Fourier transform, the gridded visibilities $\mathrm{FI}^{M}$ are interpolated from a regular grid and re-sampled at the measured $(u, v, w)$ points as:

$\boldsymbol{V}^{M^{\prime}}\left(u_{i j}, v_{i j}, w_{i j}\right)=\left(\mathbf{G}\left[\mathbf{F} \boldsymbol{I}^{M}\right]^{g}\right)\left(u_{i j}, v_{i j}, w_{i j}\right)$

where $G$ is the interpolation operator, $F$ is the Fourier transform operator and the superscript $g$ indicates data on a regular grid. The backward calculation will correspond to the application of $[\mathrm{GF}]^{\dagger}$. The operator $\mathrm{F}$ is unitary. If $\mathrm{G}$ is at least approximately unitary, $\mathrm{G}^{\dagger}$ can be used as the interpolation operator for re-sampling the data on a regular grid to correct for the effects of $\mathrm{G}$ in the image. Applying $\mathrm{G}$ and $\mathrm{G}^{\dagger}$ as part of the re-sampling operations require that both of these operators have finite support. In principle, any operator for which the inverse exists can be used, provided the inverse operator also has a finite support. However, since the inverse of an operator $\mathrm{X}$ involves $\operatorname{det}(\mathrm{X})^{-1}$, it is difficult to imagine $X^{-1}$ with a support size comparable to $X$. An approximate inverse operator with finite support for our case can be constructed by using $\mathrm{G}^{\dagger}$ for re-sampling the data (left-hand side of Eq. (9)) and then dividing the resulting image by $\operatorname{det}(F G)$.

The similarity between Eqs. (9) and (7) indicates that direction-dependent gains can be incorporated as part of the deconvolution iterations by using an efficient algorithm for the forward and backward calculations. We have chosen to use a technique similar to that used in the $\mathrm{w}$-projection algorithm to correct for the effects of non co-planar baselines (Cornwell et al. 2003). As discussed in Sect. 4.1, an approximately unitary operator $\mathrm{E}_{i j}$ can be constructed as the Fourier transform of Eq. (4). For our purpose, using $\mathrm{E}_{i j}^{\dagger}$ as the interpolation operator for gridding the visibilities on a regular grid and using FFT to invert the gridded visibilities would suffice. The accuracy of the forward calculation is proportional to the accuracy of $\mathrm{E}_{i j}$ which can, in principle, be arbitrarily precise (e.g. by accurate measurement of the antenna voltage pattern). An iterative deconvolution scheme using such transforms should ultimately drive the residual image to be noise-like, although it would seem desirable to limit the number of free parameters introduced in the process. Note 

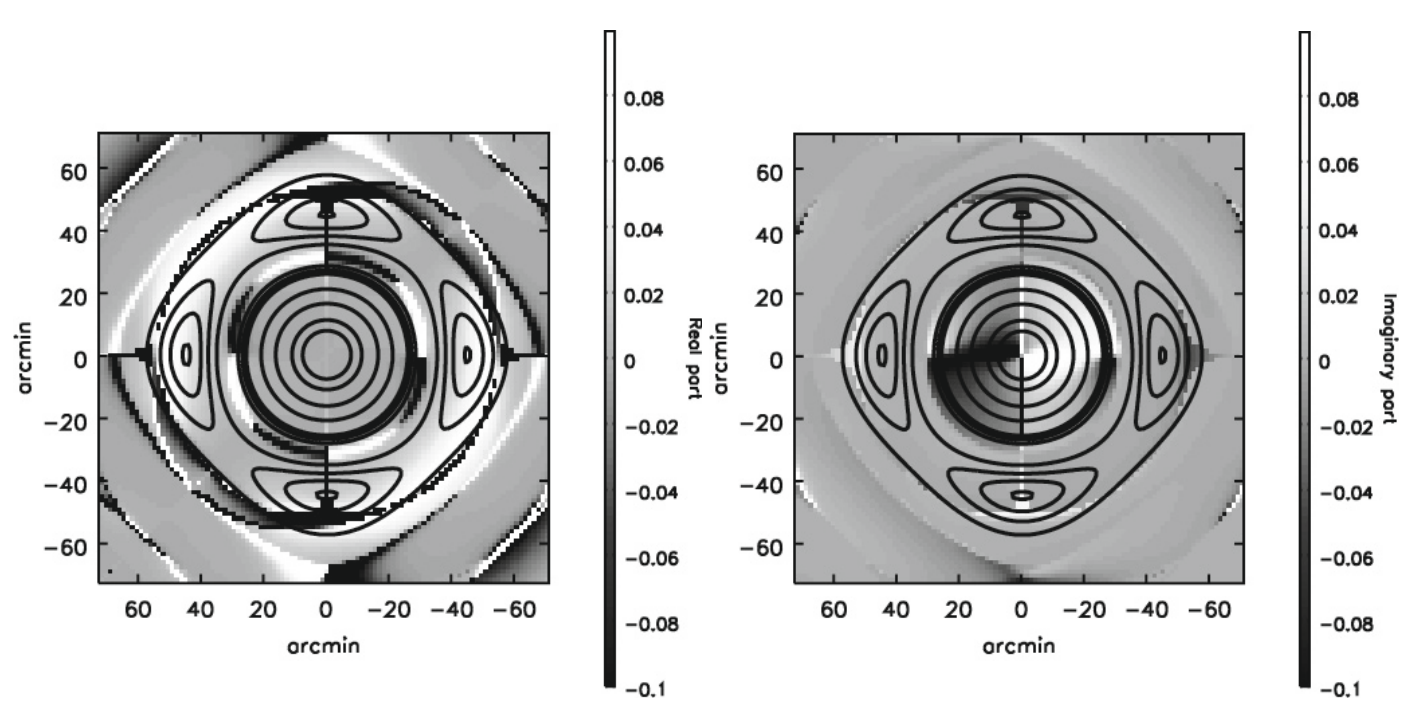

Fig. 3. The off-diagonal term of $\mathrm{J}_{i}^{\mathrm{sky}} \mathrm{J}_{i}^{\mathrm{sky}}$. Images in the left and right panels show the real and imaginary parts respectively. The overlayed contours correspond to $\max \left(J_{i}^{\text {Sky }}[0]\right) \times[0,0.1,0.15,0.18,0.2,0.4,0.6,0.8,0.9]$. The absolute maximum value inside the first null is $\sim 0.2$.

that since the final model image is iteratively built using accurate computations only in one direction, the intermediate residual dirty images have no physical meaning as is usually the case.

\subsection{Structure of the Sky Jones matrix}

Jsky affects the measurements as described by Eq. (1). The forward and backward transforms discussed above crucially depend on $\mathrm{E}_{i j}$ being at least approximately unitary. Since $\mathrm{M}_{i j}^{\mathrm{Sky}}$ is an outer product of antenna based Jones matrices (Eq. (3)) and $\mathrm{E}_{i j}=\mathcal{F} \mathcal{T}\left(\mathrm{M}_{i j}^{\text {Sky }}\right)$ where $\mathcal{F} \mathcal{T}$ represents the element-by-element Fourier transform of its argument, for our purpose it is sufficient to ensure that the $J_{i}^{\text {Sky }}$ is approximately unitary ${ }^{2}$. The diagonal terms of $\mathrm{J}_{i}^{\text {Sky }} \mathrm{J}_{i}^{\text {Sky }}$ (of the form $\mathrm{J}_{i}^{p} \mathrm{~J}_{i}^{p^{*}}+\mathrm{J}_{i}^{p q^{*}} \mathrm{~J}_{i}^{p q}$ ) correspond to the ideal (un-squinted) power patterns and are nearly equal to each other. Figure 3 shows the real and imaginary parts of the off-diagonal term normalized by $\operatorname{det}\left(\mathrm{J}_{i}^{\text {Sky }}\right)$. The peak amplitude is about two orders of magnitude lower than the diagonal term making $J_{i}$ approximately unitary. Image plane corrections therefore can be incorporated as part of the image deconvolution procedure by using $\mathrm{E}_{i j}=\mathcal{F} \mathcal{T}\left[\mathrm{M}_{i j}^{\mathrm{Sky}}\right]$ and $\mathrm{E}_{i j}^{\dagger}$ as part of the forward and reverse transforms between the visibility and image domains for baseline $i-j$.

\subsection{The overall deconvolution algorithm}

Our deconvolution algorithm proceeds as follows:

1. Initialize: Set the initial model image to zero or to a model using apriori knowledge of the sky emission (for example a model obtained with conventional techniques).

2. Major cycle:

- Forward: Compute the residual visibilities $\boldsymbol{V}^{\mathrm{Obs}}-\boldsymbol{V}^{M}$ using the observed visibilities $\boldsymbol{V}^{\mathrm{Obs}}$ for each polarization product.

- Backward: Compute the residual image using Eqs. (11) and (12) below.

\footnotetext{
${ }^{2}$ It can be shown that if $\mathrm{J}_{i}^{\text {sky }}$ is unitary, then so is $J_{i}^{\text {sky }} \otimes \mathcal{J}_{i}^{\text {sky }}$.
}

3. Minor cycle: Update the model image applying some operator $\mathrm{T}$.

4. Go to 2 until convergence is achieved, typically quantified by suitable stopping criteria (noise level, distribution of residuals, etc.).

5. Smooth the deconvolved image by the resolution element and add back the residuals.

\section{Antenna polarization and pointing error correction}

In the following section, we describe how the forward and backward calculations can account correctly for polarization leakage and pointing errors.

\subsection{Forward calculation}

In the absence of antenna pointing errors, the operator $\mathrm{E}_{i j}^{P}$ is the auto-correlation of the ideal antenna illumination patterns for polarization product $P$. It has finite support and is also approximately unitary. Therefore, it has the required properties to be used to realize the transforms necessary in a deconvolution algorithm to correct for primary beam effects. In the presence of antenna pointing errors, the operator $\mathrm{E}_{i j}^{\mathrm{P}}$ is different for each baseline $i-j$. For small pointing errors compared to the half power beam width, pointing errors contribute a linear phase gradient across the aperture. Therefore, the full $E_{i j}^{P}$ including antenna pointing errors can be efficiently evaluated by separating it into terms that include the effects which are equal for all antennas (e.g. the polarization squint of the VLA antennas) and effects which vary between antennas (e.g. the antenna-based pointing offsets) as:

$\mathrm{E}_{i j}^{\mathrm{P}}=\mathrm{E}^{\mathrm{P}^{\circ}} f\left(\phi_{i}-\phi_{j}\right) \mathrm{e}^{\iota\left(\phi_{i}+\phi_{j}\right)}$

where $\phi_{i}$ is the pointing offset for antenna $i$ and $\mathrm{E}^{\mathrm{P}^{\circ}}$ is the autocorrelation of the ideal antenna illumination pattern. The function $f$ represents the de-correlation that the signal suffers at each baseline due to the antenna pointing errors. This function is unity at the origin $(f(0)=1.0)$ and for voltage patterns with finite support, it will be a monotonically decreasing function of its 
argument (for typical illumination functions) ${ }^{3}$. However, its exact form will depend upon the actual form of the voltage patterns. For small pointing errors (few percent of the half-power beam width), it will be close to unity to the first order. When using $\mathrm{E}_{i j}^{\mathrm{P}}$ as the visibility plane filter for baseline $i-j$, with no pointing errors the predicted visibilities will correspond to a sky tapered by the corresponding power pattern (as it should be). With pointing errors, the predicted visibilities will include the effects of pointing errors.

\subsection{Backward calculation}

The backward calculation can be realized by using $\mathrm{E}_{i j}^{\mathrm{P}^{\dagger}}$ as the interpolation operator for re-sampling $\boldsymbol{V}^{\mathrm{P}}\left(u_{i j}, v_{i j}\right)$ (the visibilities for the polarization product $P$ ) on a regular grid at pixels labeled by indices $(n, m)$ as:

$\boldsymbol{V}^{\mathrm{P}, G}(n \Delta u, m \Delta v)=\left(\mathrm{E}_{i j}^{\mathrm{P}^{\dagger}} \boldsymbol{V}^{\mathrm{P}}\left(u_{i j}, v_{i j}\right)\right)(n \Delta u, m \Delta v)$

where the superscript $G$ is used to indicate a regular grid. The images corresponding to the gridded visibilities are then computed as

$\boldsymbol{I}^{d}=\operatorname{det}\left(\overline{\mathrm{F}^{\dagger}\left[\mathrm{E}^{\mathrm{P}^{\dagger}}\right]}\right)^{-1} \mathrm{~F}^{\dagger} \boldsymbol{V}^{\mathrm{P}, G}$.

The net effect of $E_{i j}$ in the image domain (expressed by $\overline{\mathrm{F}^{\dagger}\left[\mathrm{E}^{\mathrm{P}^{\dagger}}\right]}$ in Eq. (12) above) is therefore averaged over all antennas for the entire range of parallactic angle coverage.

\section{Results}

\subsection{Simulations}

The algorithm was tested for VLA squint and gain variations due to the rotation of azimuthally asymmetric antenna power patterns on the sky.

The visibilities were simulated using the $\mathcal{C A S} \mathcal{A}^{4}$ package with the parameters listed in Table 1. A model for typical sky emission at $1420 \mathrm{MHz}$ was generated using the NVSS source list. The actual rendition has 74 point sources with flux densities ranging between $195 \mathrm{mJy}$ and $2 \mathrm{mJy}$. A PA increment of $10^{\circ}$ was used in order to simulate the rotation of the R- and L-beams on the sky. The visibilities were simulated for VLA C-array and an rms noise of $\sim 1 \mathrm{mJy}$ per visibility sample was added to simulate an image plane rms noise of $\sim 1 \mu \mathrm{Jy} /$ beam. Without squint correction, the peak and rms noise in the resultant Stokes- $V$ image were $\sim 2 \mathrm{mJy}$ and $\sim 10 \mu \mathrm{Jy} /$ beam respectively. The Stokes- $V$ image generated using the algorithm described in this paper was noise-like with an $\mathrm{rms} \sim 1 \mu \mathrm{Jy} /$ beam. The squint correction results in an improvement of the noise figure by a factor of $\sim 10$. The visibilities with pointing errors were simulated by predicting the model visibilities using the forward transform (Sect. 5.1) with $\mathrm{E}_{i j}$ computed for increments of $10^{\circ}$ in PA. A model for the VLA aperture illumination pattern (Brisken 2003) was used to generate a non-azimuthally symmetric power pattern. The model includes the geometry of the sub-reflector and the feed position as well as the aperture blockage due to the feed legs and the sub-reflector. The averages of the pointing errors for each antenna were randomly distributed between $\pm 25^{\prime \prime}$ with an rms of

\footnotetext{
3 This might not be a monotonically decreasing function for all ways in which a feed might illuminate a secondary/primary reflector.

${ }^{4}$ http://casa.nrao.edu
}

Table 1. Simulation parameters.

\begin{tabular}{lc}
\hline \hline Range of parallactic angle $\left({ }^{\circ}\right)$ & $183-45$ \\
Number of visibilities & $10^{6}$ \\
Integration time $(\mathrm{s})$ & 10 \\
Number of frequency channels & 1 \\
Frequency $(\mathrm{GHz})$ & 1.4 \\
Noise per sample $(\mathrm{mJy})$ & 1 \\
Max. baseline $(\mathrm{Km})$ & 3 \\
Number of antennas & 27 \\
\hline
\end{tabular}

$5^{\prime \prime}$. The images were deconvolved using standard image deconvolution procedures and again using the above algorithm. The Stokes-I images are shown in Fig. 4. As expected, the deconvolution errors were maximal for the sources around the halfpower point and in the first sidelobe of the power pattern. These errors were eliminated when the pointing and squint correction were applied during deconvolution. The bottom panels of Fig. 4 show the Stokes- $V$ images without and with pointing and squint corrections.

\subsection{VLA 1.4 GHz data}

The algorithm was also tested for Stokes- $I$ and $-V$ imaging using VLA $1.4 \mathrm{GHz}$ observations of the superthin galaxy IC2233 (Matthews \& Uson 2008). The field contains two strong sources ( $\sim 854 \mathrm{mJy} /$ beam and $\sim 145 \mathrm{mJy} /$ beam $)$ on opposite sides of the pointing center, located at positions of $\sim 75 \%$ and $\sim 35 \%$ primary beam response levels respectively. The observations were made in spectral mode with channels of width $\sim 24 \mathrm{kHz}$ for a total of $\sim 11.6 \mathrm{~h}$ in 2 passes with well distributed uv-coverage. The line-free channels (11 from the second "IF pair") were used for the tests described here. The aperture illumination pattern for each antenna was assumed to be the same and computed using the model for VLA antennas (Brisken 2003). The aperture illuminations were computed as a function of PA in increments of $1^{\circ}$. The expected thermal noise for this data is $\sim 0.13 \mathrm{mJy} /$ beam. The results of the imaging run with and without the correction for time-varying primary beam gains and polarization squint are shown in Figs. 5 and 6. The peak negative and positive residual in the Stokes- $V$ images without primary beam correction is $-9.7 \mathrm{mJy} /$ beam and $2.8 \mathrm{mJy} /$ beam respectively. After the primary beam correction, the peaks were $\pm 0.5 \mathrm{mJy} /$ beam and uncorrelated with location of the bright sources, with an rms noise of $0.15 \mathrm{mJy} / \mathrm{beam}$.

\section{Error analysis}

Convergence of the deconvolution iterations is judged by the statistics in the residual image at convergence. Errors in the final residual image purely due to primary beam $(\mathrm{PB})$ effects (within the main-lobe) can be expressed as:

$$
\boldsymbol{I}^{R}=\sum_{\psi} P S F(\psi) \star\left[\Delta P B(\psi) \boldsymbol{I}^{\circ}\right]
$$

where " $\star$ " represents the convolution operator, $\psi$ is the feed Parallactic Angle, $\triangle P B(\psi)$ is the error between the true and the assumed primary beam model at PA $=\psi, I^{\circ}$ is the true sky distribution and $\operatorname{PSF}(\psi)$ is the instantaneous (snapshot) PSF. When imaging using an azimuthally symmetric PB model, the PB error pattern is given by $\triangle P B(\psi)=\overline{P B}-P B(\psi)$ where $\overline{P B}$ is the azimuthally averaged $\mathrm{PB}$. Rotation of this error pattern on the 

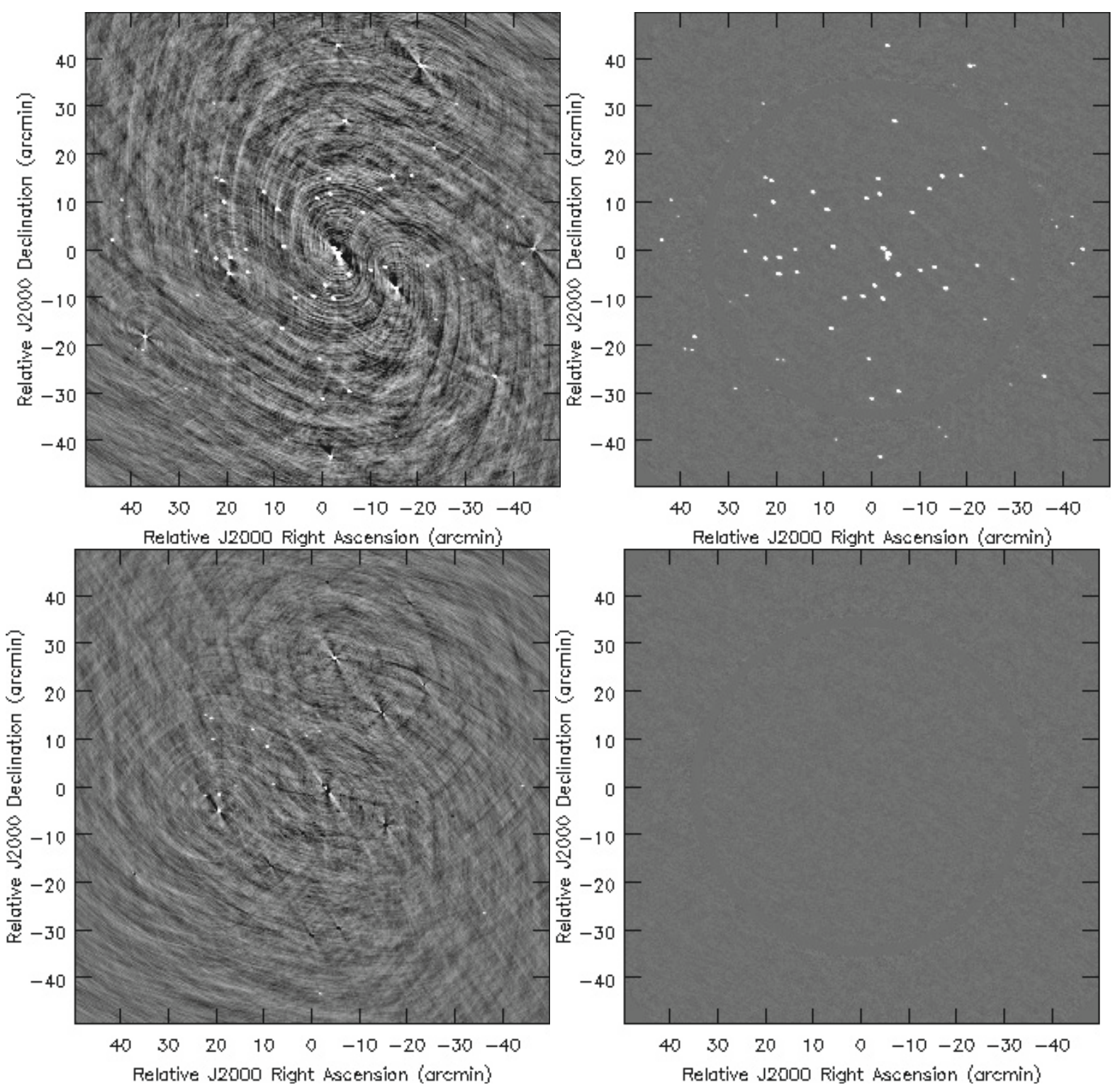

Fig. 4. The top row shows the Stokes-I images and the bottom row shows the Stokes- $V$ images. The images on the left were made without squint and pointing correction while those on the right had both corrections applied. The deconvolution errors seen around the strongest sources are due to the antenna pointing errors and time varying direction dependent gain due to the rotation of azimuthally asymmetric antenna power patterns. These images were made using a linear transfer function with the gray scales in the range $-20 \mu \mathrm{Jy} / \mathrm{beam}$ (black) and $+40 \mu \mathrm{Jy} / \mathrm{beam}(\mathrm{white})$. The rms noise in the off-source regions of the images in the left and the right panels is $10 \mu \mathrm{Jy} / \mathrm{beam}$ and $1 \mu \mathrm{Jy} / \mathrm{beam}$ respectively.

sky contributes the dominant systematic errors in the residual image (and consequently in the final deconvolved image). The peak residual can be estimated for a point source of flux density $S$ located at the position of the peak of the error pattern multiplied by the maximum sidelobe of the instantaneous PSF at $\mathrm{PA}=\psi_{i}$ :

$$
\left.I^{R}\right|_{\max }=\left[\left.P S F_{\text {sidelobe }}\left(\psi_{i}\right)\right|_{\max }\right]\left[\left.\Delta P B\left(\psi_{i}\right)\right|_{\max }\right] S .
$$

Instantaneous Stokes- $I$ and - $V$ VLA antenna power patterns at $1.4 \mathrm{GHz}$ are shown in Fig. 7. The patterns rotate on the sky with PA which results in time-varying, position-dependent gain across the field of view. The $\triangle P B$ and an azimuthal cut through this error pattern at 50\%,10\% and $1 \%$ point of $\overline{P B}$ are shown in Figs. 8 and 9 respectively. The contours correspond to $\overline{P B}_{\max } \times$ $[0.01,0.05,0.07,0.2,0.3,0.4,0.5,0.6,0.7,0.8,0.9,0.95]$. Within the main-lobe of the Stokes- $I$ beam, the peak of the error pattern is where $\overline{P B}$ is between $1-10 \%$. For $S=1 \mathrm{Jy}$,
$\left.P S F_{\text {sidelobe }}\left(\psi_{\circ}\right)\right|_{\max }=40 \%$ (measured for the test $1.4 \mathrm{GHz}, \mathrm{C}$ array data) and $\left.\triangle P B\left(\psi_{\circ}\right)\right|_{\max }=0.005$, the peak residual will be about 2 mJy in Stokes- $I$. Peak residuals in Stokes- $V$ would be at the level of about $10 \mathrm{mJy}$.

The deconvolution algorithm described above consists essentially of approximating the function shown in Fig. 9 (or equivalently the $2 \mathrm{D}$ function shown in Fig. 7) by a piece-wise constant function. The maximum error due to such an approximation can be estimated using the following equation:

$$
I_{\max }^{R}=\Delta I_{\max }^{R}[\Delta \psi]
$$

where $\Delta I_{\max }^{R}=\left.S\left[\left.P S F_{\text {sidelobe }}\right|_{\max }\right] \frac{\partial P B}{\partial \psi}\right|_{\max }$.

For a required rms noise in the image of $\eta$, the peak error due to the piece-wise constant approximation should be 3-5 times smaller than $\eta$. The minimum PA increment such that the rms 

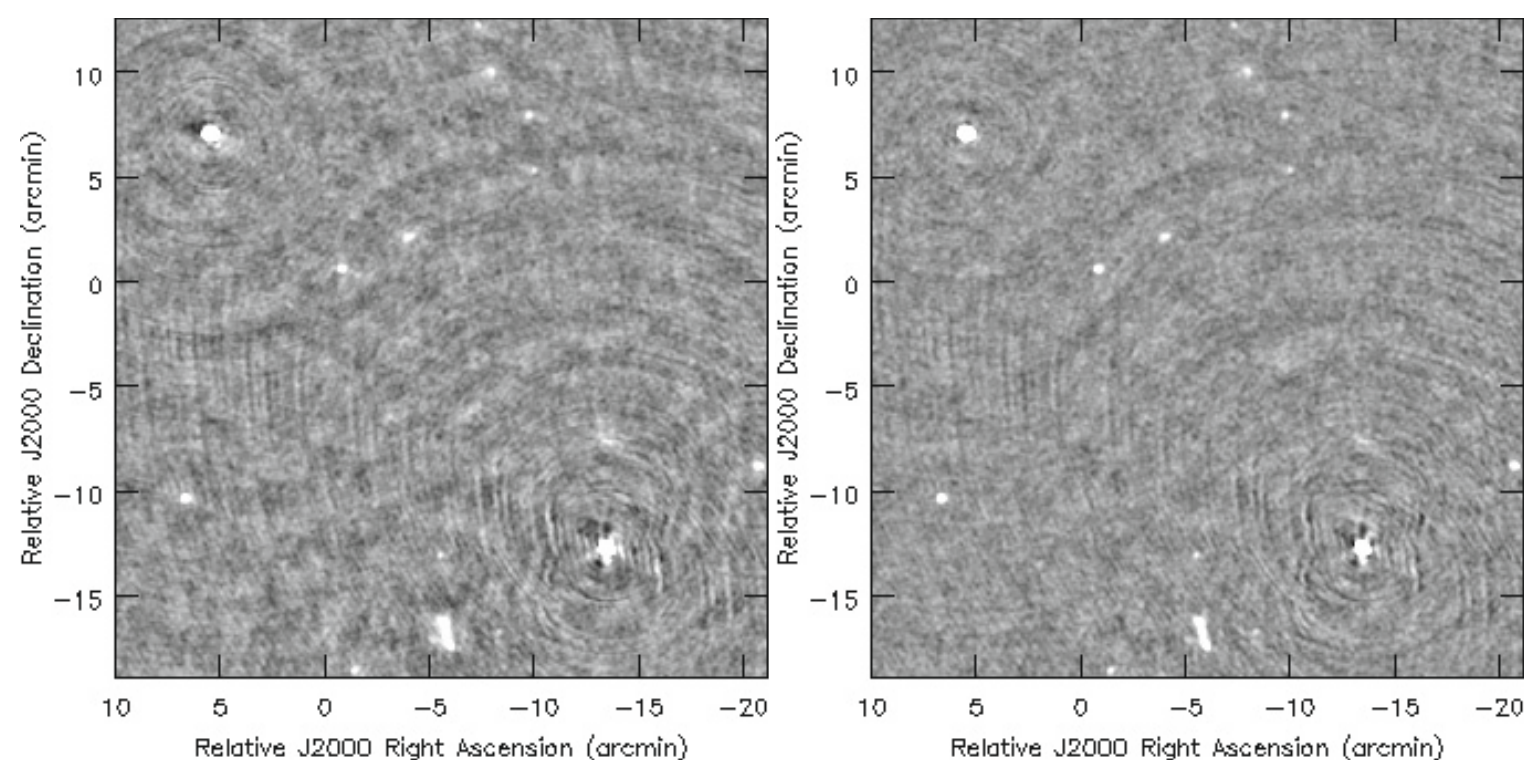

Fig. 5. The Stokes-I images at $1.4 \mathrm{GHz}$ with VLA C-array observation. The left panel shows the deconvolved image without corrections of the antenna power pattern variations as a function of parallactic angle. The right panel shows the result from the algorithm described in this paper. The two dominant sources, on either side of the pointing center have flux densities of $\sim 854 \mathrm{mJy} / \mathrm{beam}$ and $\sim 145 \mathrm{mJy} / \mathrm{beam}$ and the rms noise is $0.15 \mathrm{mJy} /$ beam. A linear transfer function with a range $\pm 1.5 \mathrm{mJy} /$ beam was used to make these images.
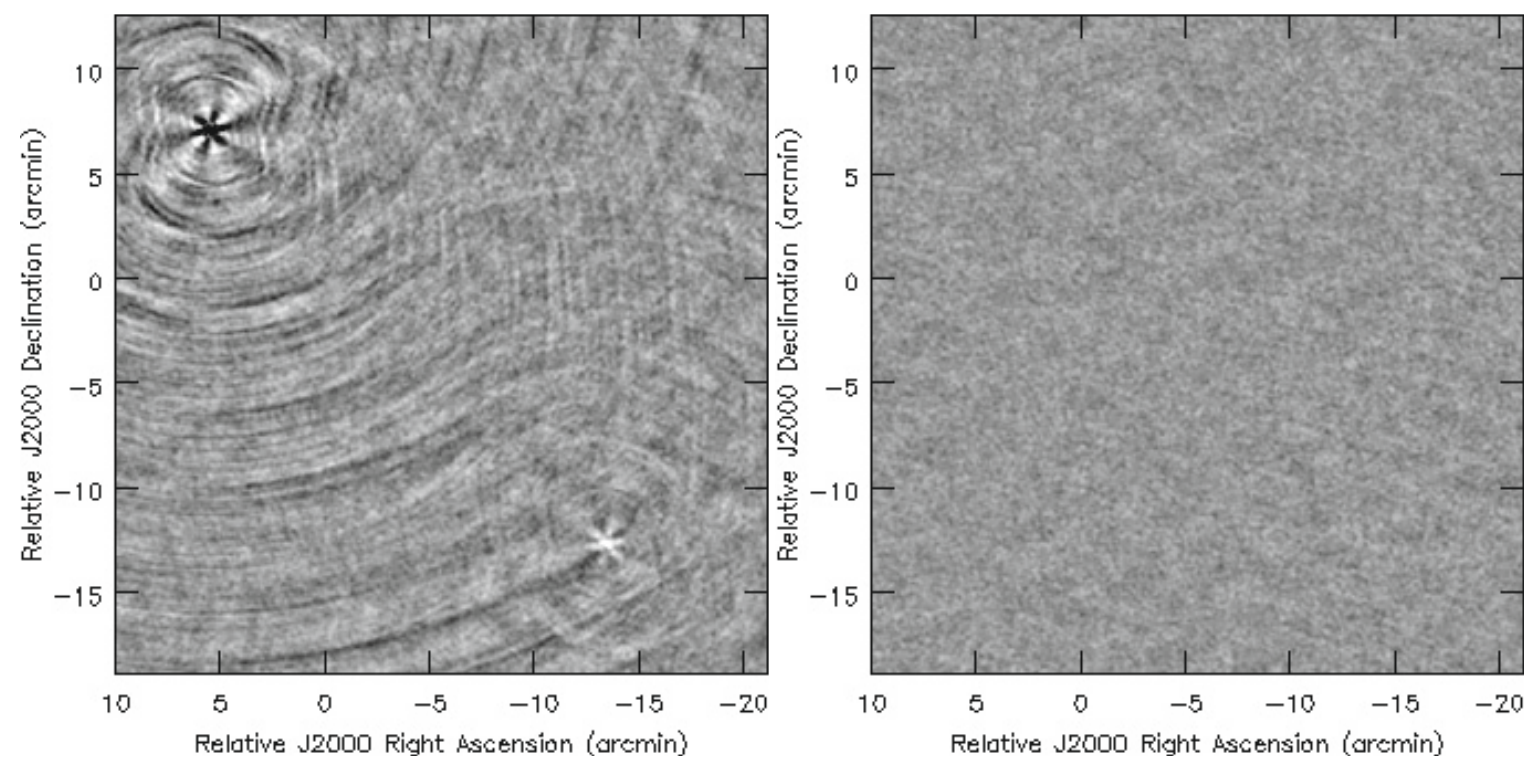

Fig. 6. The Stokes- $V$ images for a $1.4 \mathrm{GHz}$ VLA C-array data. The left and right panels show the images without and with PB-corrections. A linear transform with a range $\pm 1.5 \mathrm{mJy} / \mathrm{beam}$ was used to make these images. Errors due to the polarization squint are greater than thermal noise in the left image. The rms in the right-hand-side image is $\sim 0.15 \mathrm{mJy} / \mathrm{beam}$. The negative and positive peaks in this image are $\pm 0.5 \mathrm{mJy} / \mathrm{beam}$.

noise in the image is not limited by the piece-wise constant approximation would be given by

$\Delta \psi \leq 3 \eta / \Delta I_{\max }^{R}$.

For the $1.4 \mathrm{GHz}$, VLA C-array test data, $\left.\frac{\partial P B}{\partial \psi}\right|_{\max } \approx$ $0.0003 \mathrm{deg}^{-1}$. For a PA increment of $10^{\circ}$, peak residuals in Stokes- $I$ and $-V$ would be about $1 \mathrm{mJy}$ and 5 mJy respectively. With the expected thermal sensitivity of $0.1 \mathrm{mJy} /$ beam for the $1.4 \mathrm{GHz}$ test data we used, PA increments of $1^{\circ}$ were required.

The PA increment for higher sensitivity telescopes like the EVLA or SKA such that imaging is not limited by the above approximation will be much smaller. This requirement however can be significantly relaxed by approximating the error function by a piecewise linear approximation (interpolation of the functions computed at larger PA increments). Furthermore, since image interpolation itself can be expensive, caching of precomputed aperture functions at appropriate PA increments will be necessary. Note that the gridding cost is relatively insensitive to the number of convolution functions used. A hybrid approach of FFT based transforms plus analytical computations for the strongest sources will probably deliver optimal performance.

\section{Discussion}

Some residual deconvolution errors are still left around the second strongest source in Fig. 5. The pattern in the residual image (not shown) suggests that these errors are due to image pixelation (Voronkov \& Wieringa 2004; Cotton \& Uson 2007). 


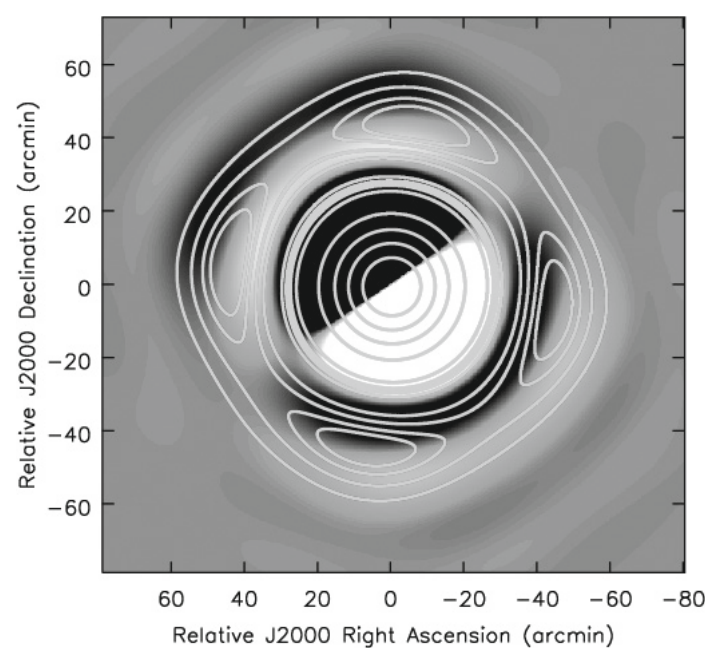

Fig. 7. Model for the VLA $1.4 \mathrm{GHz}$ antenna at Parallactic Angle $\sim 80^{\circ}$. The Stokes- $V$ pattern $\left(\left[P B_{R R}-P B_{L L}\right] / 2\right)$ is shown in colour/gray scale with the contours of the Stokes- $I$ power pattern superimposed. Dark regions in the gray scale image represent negative values due to the polarization squint of VLA antenna.

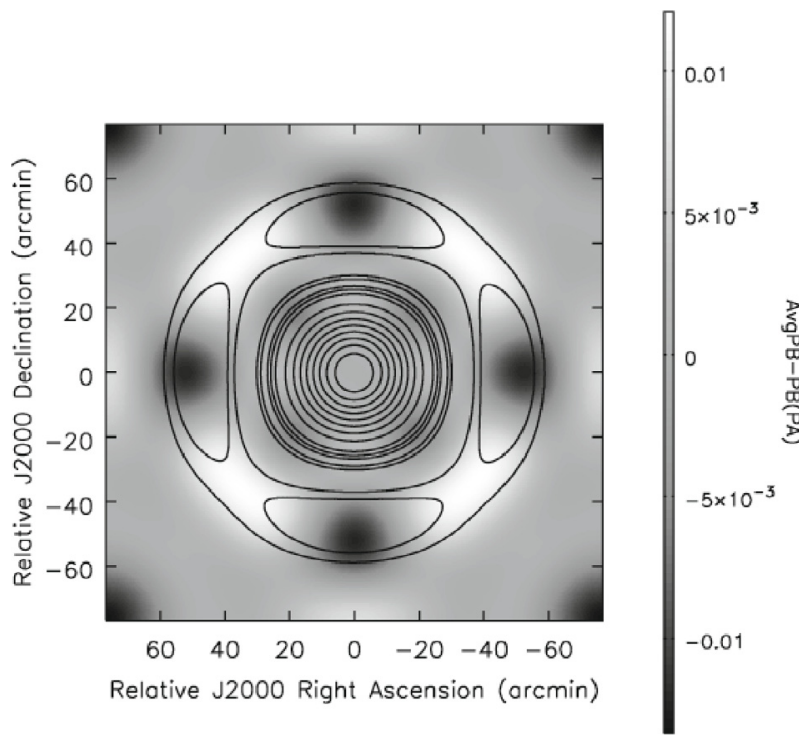

Fig. 8. Difference between an instantaneous Stokes- $I$ PB and an azimuthally averaged $\mathrm{PB}(\overline{P B})$. The colour/gray scale image is $\triangle P B=$ $P B\left(\psi_{\circ}\right)-\overline{P B}$ while the contours are for the $\overline{P B}$.

More sophisticated parametrization of the sky, independent of the image pixel size (e.g. as is done in scale sensitive deconvolution algorithms like the Asp-Clean (Bhatnagar \& Cornwell 2004) or MS-Clean) along with the imaging algorithm described here to correct for DD gains should give better results. It is also possible that the residual errors are due to pointing errors during the observation. We are investigating this possibility and hope to report on it in due course.

\subsection{Why not peeling?}

The algorithm described here corrects for DD gains without loosing the efficiency advantage of the FFT algorithm. Our algorithm scales well in run-time efficiency and implementation complexity for large data volume, complex field as well as for

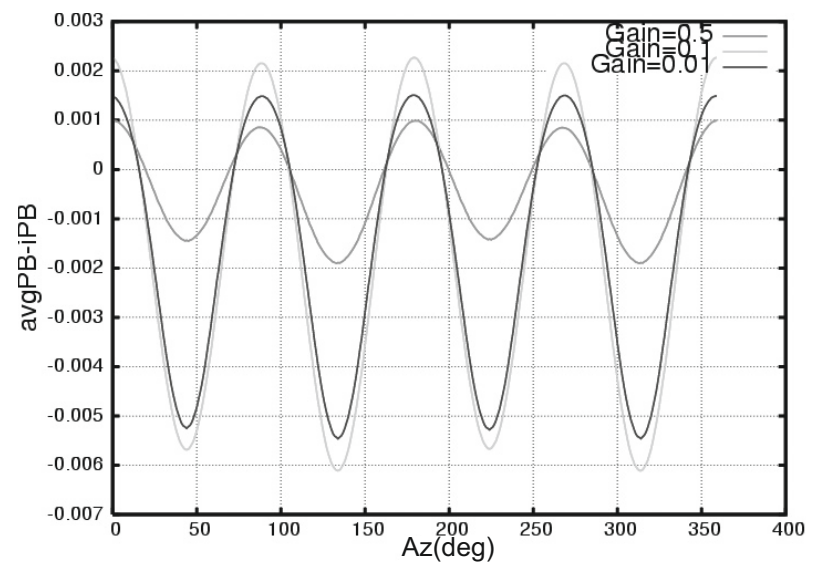

Fig. 9. Azimuthal cut through $\triangle P B$ shown in Fig. 8 at points where the value of the $\overline{P B}$ is at $50 \%, 10 \%$ and $1 \%$ of its peak value.

arrays where antenna elements cannot be assumed to be identical.

Various variants of the "Peeling" algorithm can also be used to correct for direction-dependent gains. In this approach antenna based gains are determined in the direction of each compact source. These gains are then used to subtract the contribution of compact sources from the observed data using a Direct Fourier Transform (DFT) and the residual visibilities are imaged again. While this is useful in removing the artifacts due to strong compact sources, since the gains are determined independently for each direction in the sky, as the image complexity increases, too many degrees of freedom (d.o.f.) might be added to the problem. For crowded fields (large number of compact sources), this leads to a proliferation of d.o.f.s and potentially to the problem of over-fitting (the extreme case being when each pixel in the image has an associated independent gain which gives the bestfit result). Since DFTs have to be used to compute residuals, the computing load is also significantly higher than the corresponding one for FFT-based computation of residuals. For complex fields containing extended emission, this approach quickly becomes numerically un-viable because of the large number of d.o.f.s included as well as the high computing and I/O loads involved. Therefore, while variants of the Peeling algorithm could have given better results for the particular $1.4 \mathrm{GHz}$ VLA data that we have used in this paper, we did not resort to Peeling based algorithms. However since the goal here is to demonstrate the effectiveness of the algorithm in correcting for otherwise difficult to correct DD gains, we used this relatively simple field for our tests so that the advantages and limitations of our algorithm are brought to the fore.

Of course, using a direct Fourier transform (DFT) for predicting model visibilities rather than using the FFT algorithm will give the most accurate results. While such a brute-force approach might be useful for simple fields, as mentioned above, the computing cost for even such simple fields becomes prohibitive for data with more than a few frequency channels, even when assuming that the various antenna elements are identical. For cases where this assumption breaks down, as it does even for the simple case of random antenna pointing errors, computing costs are impractically high. Furthermore, for full-beam, full-Stokes imaging, which requires use of at least the diagonal terms of the Mueller matrix (Eq. (4)) if not the full matrix, it is unclear if a brute-force DFT approach will work. 


\subsection{Implications for wideband and mosaic imaging}

Antenna pointing errors, azimuthally asymmetric aperture illuminations, wide bandwidths and deconvolution errors due to the use of discrete pixels for the sky representation all leave residuals that limit the full-beam imaging dynamic range to $10^{4}-10^{5}$. Therefore, apart from correcting for the direction dependent effects, for the highest imaging dynamic range, scale-sensitive decomposition of the sky might also be necessary (Bhatnagar \& Cornwell 2004). The algorithm described here can be combined efficiently with scale-sensitive deconvolution and has the potential of overcoming the above mentioned imaging dynamic range limit.

The algorithm described here accounts for the time varying gain variations due to the rotation of the azimuthally asymmetric aperture illumination with PA. For imaging with a large bandwidth ratio (e.g. the ratio of frequencies at the two edges of the observing band for EVLA will be 2:1), the dominant error term will be the scaling of the power pattern with frequency. Sources which will be well within the main lobe of the primary beam at the lower frequency end of the band will be outside the main lobe at the higher frequency end (and may even appear in the first sidelobe). Since the azimuthal variations in the power pattern due to feed-leg/sub-reflector blockage are maximal close to the null and in the first sidelobe, frequency scaling of the aperture illumination will contribute a first-order error.

Scaling of the antenna power patterns with frequency in observations with wide bandwidths can be incorporated in the algorithm described here by computing the aperture illumination functions at appropriate increments in frequency. Alternatively, depending on the required accuracy, this scaling can be achieved as well by scaling the co-ordinates with frequency. Since the computing cost scales weakly with the number of convolution functions used, the extra computing load will not be too high. The cost of computing the aperture functions itself will be significant, but it is a one-time cost.

Rotation of the sidelobes results in gain variations on the order of a factor of two in the direction of the sidelobes. For mosaic observations, this will contribute significant time-varying flux density in most individual pointings; assuming a peak PSF sidelobe of $10 \%$, the error inside the main lobe of the power pattern will be at the level of a few percent of the peak flux in the direction of the first sidelobe of the antenna power pattern. This will limit the mosaicking dynamic range significantly, indeed it will be a first-order effect. In addition, a second-order effect will be due to antenna pointing errors. Correction of both of these effects will be required for mosaicking instruments presently under construction like the ALMA and the ASKAP (Johnston 2007). The general framework and the algorithm described here can be generalized easily for application to mosaic imaging and could correct errors due to the rotation of antenna primary beams as well as pointing errors. In practice however, the imaging dynamic range might be limited by the precision with which antenna power patterns and pointing errors can be determined. Furthermore, a similar approach can be used for imaging with inhomogeneous arrays like CARMA/ALMA (where not all antennas in the array are identical) as well as with arrays with multi-feed antennas like the ASKAP. Finally, for very high dynamic range imaging with telescopes like LOFAR, SKA, and even EVLA, nominally identical antenna elements may have variations that will induce errors higher than the thermal noise limit. In that sense, such telescopes will also need to be treated as inhomogeneous arrays.

\section{Conclusions}

Existing imaging algorithms ignore the effects of time varying gains due to antenna pointing errors and rotation of azimuthally asymmetric antenna power patterns. As shown in Sects. 6 and 7 using the VLA as an example, residual errors due to these effects are maximal in the first sidelobe and significant even within the main lobe of the antenna power pattern. Simulations show that the errors due to these effects limit the achievable dynamic range for sensitive radio interferometers under construction like the EVLA, ALMA and ASKAP. The full polarimetric response of the antenna is also inherently asymmetric due to the physics of reflection from curved surfaces. In addition, the voltage patterns of the two orthogonal polarizations for the VLA antennas are separated on the sky (polarization squint) resulting in increasing instrumental Stokes- $V$ as a function of distance from the image center. Therefore, for moderate dynamic range full-beam, full-Stokes imaging, the Sky Jones matrix for the VLA antennas cannot be assumed to be scaled-identity or even diagonal. Even for antennas without polarization squint, the off-diagonal terms will remain significant, even though the difference between the parallel-hand terms may be negligible. Hence, full-Sky Jones matrix treatment is necessary for full Stokes imaging of most observed fields. This implies a four-fold increase in the computing load when compared to imaging when primary beam effects are neglected.

The deconvolution algorithm described in this paper corrects for systematic effects due to non-ideal primary beams by modeling the complex antenna aperture illumination for the two orthogonal polarizations as a function of parallactic angle and antenna pointing errors. The antenna aperture functions are used to construct precise forward and approximate inverse transforms, exploiting the property that the Sky Jones matrix is approximately unitary. We have applied this algorithm to VLA $1.4 \mathrm{GHz}$ imaging and show that the instrumental Stokes- $V$ is eliminated to an accuracy of better than $10 \%$, possibly limited by uncertainties in our model of the primary beam as well as pointing errors that were not corrected in this reduction because our algorithm does not yet handle pointing errors and selfcalibration simultaneously. Simulations of single pointing observations at $1.4 \mathrm{GHz}$ with the EVLA with typical time-varying antenna pointing errors show that antenna pointing errors limit the imaging dynamic range at a level of $\sim 10^{5}: 1$ away from strong sources in a typical field (imaging dynamic range could be even lower if you are unlucky, like for the IC2233 case). Using this algorithm on this simulated data we demonstrate that the effects of antenna pointing errors can also be corrected during deconvolution. This approach can therefore be used for full-beam full-Stokes imaging.

Finally, we note that in the presence of image plane errors, imaging and calibration algorithms are more tightly coupled compared to those appropriate to direction-independent calibration. Solvers for parameters which describe direction dependent errors require the forward transform used during imaging. Correction for direction dependent effects is done during image deconvolution and one cannot produce corrected visibilities independent of full image deconvolution. With the advent of higher sensitivity arrays where many direction dependent errors will need to be accounted for, modern imaging and calibration software must be designed to easily accommodate these cases.

Acknowledgements. All of this work was done using the $C \mathcal{A S} \mathcal{A}$ package. We thank W. Brisken for his help in modeling the VLA antenna aperture function and M. A. Voronkov for his many useful comments. We thank Lynn Matthews for her excellent bandpass calibration of the IC 2233 data. We have benefited from discussions with Bill Cotton, Rick Fisher, Rick Perley and Ken Sowinski. 


\section{References}

Andrews, H. C., \& Hunt, B. R. 1977, Digital Image Restoration (New York: Prentice Hall)

Bhatnagar, S., \& Cornwell, T. J. 2004, A\&A, 426, 747

Brisken, W. 2003, Using Grasp8 To Study The VLA Beam, Tech. rep., EVLA Memo 58

Cornwell, T. J. 1988, A\&A, 202, 316

Cornwell, T. J., Golap, K., \& Bhatnagar, S. 2003, W-Projection: A new algorithm for non-coplanar baselines, Tech. rep., EVLA Memo, 67

Cotton, W. D. 1999, in Synthesis Imaging in Radio Astronomy II, ed. R. A. Perley, F. R. Schwab, \& A. H. Bridle, ASP Conf. Ser., 6, 233

Cotton, W. D., \& Uson, J. M. 2007, EVLA Memo 114

EVLA Memo 114
Hamaker, J. P., Bregman, J. D., \& Sault, R. J. 1996, A\&AS, 117, 137

Högbom, J. A. 1974, A\&AS, 15, 417

Johnston, S. 2007, arXiv e-prints, 711

Jones, R. C. 1941, J. Opt. Soc. Amer., 31, 488

Kraus, J. D. 1986, Radio astronomy (Powell, Ohio: Cygnus-Quasar Books)

Matthews, L. D., \& Uson, J. M. 2008, AJ, 135, 291

Mueller, H. 1948, J. Opt. Soc. America, 38, 661

Schwab, F. R. 1983, in Indirect Imaging, ed. J. A. Roberts (Cambridge, England:

Cambridge University Press), 333

Schwarz, U. J. 1978, A\&A, 65, 345

Voronkov, M. A., \& Wieringa, M. H. 2004, Exper. Astron., 18, 13 